\title{
Five-frequency Galileo long-baseline ambiguity resolution with multipath mitigation
}

\author{
Kan Wang ${ }^{1}$ Amir Khodabandeh ${ }^{1,2} \cdot$ Peter J. G. Teunissen ${ }^{1,3}$
}

Received: 11 October 2017 / Accepted: 8 May 2018 / Published online: 22 May 2018

(c) The Author(s) 2018

\begin{abstract}
For long-baseline over several hundreds of kilometers, the ionospheric delays that cannot be fully removed by differencing observations between receivers hampers rapid ambiguity resolution. Compared with forming ionospheric-free linear combination using dual- or triple-frequency observations, estimating ionospheric delays using uncombined observations keeps all the information of the observations and allows extension of the strategy to any number of frequencies. As the number of frequencies has increased for the various GNSSs, it is possible to study long-baseline ambiguity resolution performance using up to five frequencies with uncombined observations. We make use of real Galileo observations on five frequencies with a sampling interval of $1 \mathrm{~s}$. Two long baselines continuously receiving signals from six Galileo satellites during corresponding test time intervals were processed to study the formal and empirical ambiguity success rates in case of full ambiguity resolution (FAR). The multipath effects are mitigated using the measurements of another day when the constellation repeats. Compared to the results using multipath-uncorrected Galileo observations, it is found that the multipath mitigation plays an important role in improving the empirical ambiguity success rates. A high number of frequencies are also found to be helpful to achieve high ambiguity success rate within a short time. Using multipath-uncorrected observations on two, three, four and five frequencies, the mean empirical success rates are found to be about $73,88,91$, and $95 \%$ at $10 \mathrm{~s}$, respectively, while the values are increased to higher than $86,95,98$, and $99 \%$ after mitigating the multipath effects.
\end{abstract}

Keywords Ambiguity resolution $\cdot$ Long-baseline $\cdot$ Galileo $\cdot$ Five frequencies

\section{Introduction}

In relative positioning using the Global Navigation Satellite System (GNSS), precise positioning results with accuracy of millimeter to centimeter can be achieved after correctly resolving the phase ambiguities. Depending on the ionospheric activities, for baselines with lengths up to 10-20 km, the atmospheric delays, i.e., the tropospheric delays and the ionospheric delays, can be significantly reduced by forming single differencing observations between receivers. The phase ambiguities can thus be resolved within short

Peter J. G. Teunissen

p.j.g.teunissen@tudelft.nl

1 GNSS Research Centre, Curtin University, GPO Box U1987, Perth, WA 6845, Australia

2 School of Civil and Environmental Engineering, University of New South Wales, Sydney, NSW 2052, Australia

3 Department of Geoscience and Remote Sensing, Delft University of Technology, 2628 CN Delft, The Netherlands convergence time, or even in one epoch only (Tiberius et al. 2002). For longer baselines, however, the remaining differential atmospheric delays may strongly hinder successful integer ambiguity resolution (IAR).

Forming the ionospheric-free linear combination removes the first-order term of the ionospheric delays, which accounts for about $99 \%$ of the total ionospheric delays (Elmas et al. 2011). However, the ionospheric-free linear combination has the drawback that it has less flexibility to further strengthen the model, e.g., to constrain the temporal or spatial ionospheric behaviors (Mervart et al. 2013; Teunissen and Khodabandeh 2015). Furthermore, as stated in Teunissen and Odijk (2003), not all the ionospheric-free linear combinations could preserve the integer property of the ambiguities. In the GPS triple-frequency case, e.g., only a particular subset of the ionospheric-free linear combinations allow the parametrization of integer ambiguities. One can therefore alternatively estimate the ionospheric delays using uncombined GNSS observations. Processing based on uncombined observations keeps all the information in the 
observation equations and is easy to be extended to any number of frequencies (Odijk et al. 2016). In the dual-frequency case, however, this model is too weak for fast ambiguity resolution (Odijk et al. 2014a). Applying ionospheric constraint based on a regional ionospheric model during ionospheric quiet days, according to Zhang et al. (2017), can accelerate the ambiguity resolution from about $18 \mathrm{~min}$ to about $5 \mathrm{~min}$ on average using 30-s dual-frequency GPS observations for baselines with an average distance of around $95 \mathrm{~km}$.

With the fast development of various GNSS during the last ten years, it is possible to exploit the benefits of the increasing number of frequencies on long-baseline ambiguity resolution. Diverse studies have been performed based on simulated multi-frequency GNSS signals. Jonkman et al. (2000) investigated the impact of a third GPS frequency on long-baseline ambiguity resolution based on the ambiguity resolution success rates using the geometry-free model. It was found that the third frequency helps to achieve substantial reduction in time for reliably fixing the ambiguities. Using simulated $1 \mathrm{~Hz}$ triple-frequency GPS and Galileo data, according to Zhang et al. (2003), it takes about 70 and $35 \mathrm{~s}$ on average to correctly fix the ambiguities for baseline of $50 \mathrm{~km}$ in GPS-only and Galileo-only triple-frequency cases, respectively, with the mean Time-To-Fix-Ambiguities (TTFA) rapidly increasing with the baseline length. Based on a hardware simulation, Sauer et al. (2004) have shown a mean TTFA of around 85, 60 , and $50 \mathrm{~s}$ using two, three, and four frequencies of simulated Galileo $1 \mathrm{~Hz}$ data for a $85 \mathrm{~km}$ baseline, respectively, with the TTFA increasing with the baseline length. Using triple-frequency GPS real/semi-generated observations for baselines over $200 \mathrm{~km}$, with an ionosphere-weighted model applied, the IAR time was found to be around 20-25 min using $30 \mathrm{~s}$ data (Ning et al. 2016). Apart from these investigations, studies were also performed for long-baseline ambiguity resolution using uncombined GNSS observations. Using uncombined observations when solving for ambiguities with cascade ambiguity resolution (CAS), over $39 \mathrm{~s}$ is required to resolve the ambiguities on average for baselines longer than $250 \mathrm{~km}$ using simulated Galileo $1 \mathrm{~Hz}$ data on four frequencies (Ji et al. 2013). Odijk et al. (2014a) have performed a study to predict the success rates of long-baseline GPS and Galileo ambiguity resolution using uncombined observations with simulated receiver and satellite geometries on three frequencies. Using triple-frequency Galileo-only signals on E1, $\mathrm{E} 5 \mathrm{a}$, and E5b, the formal TTFA is found to be around 20 and 10 min using 30- and 10-s data, respectively. Making use of ionospheric modeling such as single layer model (Schaer 1999) and simulated Galileo signals, the Galileo-only triplefrequency test case with 5 -s data requires about $5 \mathrm{~min}$ for $500 \mathrm{~km}$ ground-based single-baseline to resolve ambiguities (Nardo et al. 2016). Despite different underlying models and sampling rates of the data, the phase ambiguities of medium and long baselines are mostly reported to be resolved in less than or about 60 epochs on average using simulated Galileo signals on three or four frequencies.

The European Galileo system is providing data on five frequencies for 15 operational In-Orbit Validation (IOV) and Full Operational Capability (FOC) satellites by the end of September 2017 (Galileo Constellation 2017). Instead of using simulated signals, it is now possible to study and compare the long-baseline ambiguity resolution performance using real Galileo data on two, three, four, and all five frequencies. In this contribution, undifferenced and uncombined observations are used with the slant ionospheric delays estimated as spatially and temporally unlinked parameters, so that the processing does not set conditions for the ionospheric activity. The observation model is easy to be extended to any number of frequencies. The real Galileo observations used in this study are influenced by code and phase multipath effects. Making use of Galileo observations of another day, the procedure to compute the multipath corrections and to mitigate the multipath effects is presented. The ambiguity success rates (SRs) of two long baselines consisting of continuously operating reference stations (CORS) without and with the multipath mitigation are analyzed and compared using different number of frequencies and at different processing times. We remark that the approach proposed in this contribution is applicable to CORS network ambiguity resolution with known coordinates, where the rover positioning is not performed.

In the subsequent section, we first introduce the strategy of the full integer ambiguity resolution using the LeastSquares Ambiguity Decorrelation Adjustment (LAMBDA) method (Teunissen 1993, 1995). After that, the data selection and the process for multipath mitigation are explained. The formal and empirical success rates are then analyzed and compared using two, three, four, and five Galileo frequencies. The results are discussed and concluded.

\section{Processing strategy}

In this contribution, multi-frequency Galileo-only signals are used in the observation model. The undifferenced and uncombined observed-minus-computed $(\mathrm{O}-\mathrm{C})$ terms of the multi-frequency single-system GNSS phase $\left(\Delta \phi_{\mathrm{r}, j}^{\mathrm{s}}\right)$ and code observations $\left(\Delta p_{r, j}^{s}\right)$ at the time point $t_{i}$ can be formulated as (Hofmann-Wellenhof et al. 2008; Teunissen and Montenbruck 2017)

$E\left(\Delta \phi_{r, j}^{s}\right)=g_{r}^{s} \tau_{r}+\mathrm{d} t_{r}-\mathrm{d} t^{s}-\mu_{j} l_{r}^{s}+\delta_{r, j}-\delta_{, j}^{s}+\lambda_{j} a_{r, j}^{s}+\left[m_{\phi_{r, j}}^{s}\right]$

$E\left(\Delta p_{r, j}^{s}\right)=g_{r}^{s} \tau_{r}+\mathrm{d} t_{r}-\mathrm{d} t^{s}+\mu_{j} l_{r}^{s}+\mathrm{d}_{r, j}-\mathrm{d}_{j}^{s}+\left[m_{p_{r, j}}^{s}\right]$ 
where the subscripts $r$ and $j$ represent receiver $r$ and frequency $j$, respectively, and the superscript $s$ represents satellite $s$. The zenith tropospheric delay (ZTD) for receiver $r$ (after removing the a priori value) and its mapping function for receiver $r$ and satellite $s$ are represented by $\tau_{r}$ and $g_{r}^{s}$, respectively. The receiver and the satellite clocks are denoted by $\mathrm{d} t_{r}$ and $\mathrm{d} t^{s}$, respectively, and the ionospheric delay $l_{r}^{s}$ between receiver $r$ and satellite $s$ on the reference frequency $f_{1}$ is multiplied with the factor $\mu_{j}=f_{1}^{2} / f_{j}^{2} \cdot \delta_{r, j}$ and $\delta_{j}^{s}$ stand for the receiver and satellite phase hardware biases, respectively, and $d_{r, j}$ and $d_{, j}^{s}$ denote those for code observations, respectively. The phase ambiguity is represented by $a_{r, j}^{s}$, and the corresponding wavelength is denoted by $\lambda_{j} . m_{\phi_{r, j}}^{s}$ and $m_{p_{r, j}}^{s}$ in [.] represent the phase and the code multipath effects, which will be discussed later in the paper. $E(\cdot)$ denotes expectation operator. The subscript 1 and the superscript 1 represent the reference station and satellite, respectively.

During the processing, the precise Galileo satellite orbits of the Multi-GNSS Experiment (MGEX) (MGEX 2017; Montenbruck et al. 2014, 2017) calculated by CNES/CLS/ GRGS and German Research Centre for Geosciences are also used as known parameters. As to the receiver phase center offsets (PCOs) and phase center variations (PCVs), the ones in igs08.atx (Montenbruck et al. 2015) for GPS L1 are used for Galileo E1 and those for GPS L2 are used for the other Galileo frequencies. The a priori ZTDs are computed based on the Saastamoinen model (Saastamoinen 1972), and the Ifadis mapping function (Ifadis 1986) is used as the troposphere mapping function. Since this study concentrates on the ambiguity resolution performance of CORS using signals on different number of frequencies, the coordinates of the stations provided by Geoscience Australia (GA, Geoscience Australia 2017) are assumed to be known. With the strong model with known baselines, we show the ambiguity success rates that can be achieved for Galileo long-baseline processing without and with multipath mitigation, as well as the rate changes with increasing number of frequencies.

In order to solve the rank deficiencies in (1) and (2), a set of $S$-basis parameters are constrained (Teunissen et al. 2010), so that estimable parameters are formed and a fullrank design matrix is generated. After reformulation, the $\mathrm{O}-\mathrm{C}$ terms of the phase and the code observations are represented by

$$
E\left(\Delta \phi_{r, j}^{s}\right)=g_{r}^{s} \tau_{r}+\mathrm{d} \tilde{t}_{r}-\mathrm{d} \tilde{t}^{s}-\mu_{j} \tilde{l}_{r}^{s}+\tilde{\delta}_{r, j}-\tilde{\delta}_{j}^{s}+\lambda_{j} \tilde{a}_{r, j}^{s}+\left[m_{\phi_{1, j}}^{1 s}\right]
$$

$E\left(\Delta p_{r, j}^{s}\right)=g_{r}^{s} \tau_{r}+\mathrm{d} \tilde{t}_{r}-\mathrm{d} \tilde{t}^{s}+\mu_{j} \tilde{l}_{r}^{s}+\tilde{d}_{r, j}-\tilde{d}_{j}^{s}+\left[m_{p_{1, j}}^{1 s}\right]$

where the double-differenced phase and code multipath $m_{\phi_{1 r, j}}^{1 s}$ and $m_{p_{1 r j}}^{1 s}$ in [.] remain as unmodeled effects, and the estimable parameters are listed in Table 1 with $\bar{d}_{r \neq 1, j=1,2}\left(t_{i}\right)$ and $\bar{d}_{, j=1,2}^{s}\left(t_{i}\right)$ formulated as

$\bar{d}_{r \neq 1, j=1,2}\left(t_{i}\right)=d_{r, j}\left(t_{i}\right)+m_{p_{1 r, j}}^{1}\left(t_{i}\right)$

$\bar{d}_{, j=1,2}^{s}\left(t_{i}\right)=d_{, j}^{s}\left(t_{i}\right)-m_{p_{1, j}}^{s}\left(t_{i}\right)$

$(\cdot)_{\mathrm{IF}}$ and $(\cdot)_{\mathrm{GF}}$ in Table 1 represent the ionospheric-free and geometry-free combination of the contents in $(\cdot)$, respectively, and are defined as follows:

$$
\begin{gathered}
(\cdot)_{\mathrm{IF}}=\frac{\mu_{2}(\cdot)_{, 1}-\mu_{1}(\cdot)_{, 2}}{\mu_{2}-\mu_{1}} \\
(\cdot)_{\mathrm{GF}}=\frac{(\cdot)_{, 2}-(\cdot)_{, 1}}{\mu_{2}-\mu_{1}}
\end{gathered}
$$

Using the Curtin PPP-RTK Network Software, the estimable parameters listed in Table 1 are processed in a Kalman
Table 1 Estimable parameters and $S$-basis parameters in (3) and (4) with unlinked ionospheric delays and hardware biases and linked ZTDs

\begin{tabular}{ll}
\hline Parameter & Interpretation \\
\hline $\mathrm{d} \tilde{t}_{r \neq 1}\left(t_{i}\right)$ & $\mathrm{d} t_{1 r}\left(t_{i}\right)+d_{1 r, \mathrm{IF}}\left(t_{i}\right)$ \\
$\mathrm{d} \tilde{t}^{s}\left(t_{i}\right)$ & $\mathrm{d} t^{s}\left(t_{i}\right)+d_{, \mathrm{IF}}^{s}\left(t_{i}\right)-\left(\mathrm{d} t_{1}\left(t_{i}\right)+d_{1, \mathrm{IF}}\left(t_{i}\right)\right)$ \\
$\tilde{l}_{r}^{s}\left(t_{i}\right)$ & $l_{r}^{s}\left(t_{i}\right)+d_{r, \mathrm{GF}}\left(t_{i}\right)-d_{\mathrm{GF}}^{s}\left(t_{i}\right)$ \\
$\tilde{\delta}_{r \neq 1, j}\left(t_{i}\right)$ & $\delta_{1, j}\left(t_{i}\right)+\mu_{j} d_{1 r, \mathrm{GF}}\left(t_{i}\right)-d_{1 r, \mathrm{FF}}\left(t_{i}\right)+\lambda_{j} a_{1, j}^{1}+m_{\phi_{1, j}}^{1}\left(t_{i}\right)$ \\
$\tilde{\delta}_{j}^{s}\left(t_{i}\right)$ & $\delta_{j}^{s}\left(t_{i}\right)+\mu_{j}\left(d_{\mathrm{GF}}^{s}\left(t_{i}\right)-d_{1, \mathrm{GF}}\left(t_{i}\right)\right)-\left(d_{\mathrm{IF}}^{s}\left(t_{i}\right)-d_{1, \mathrm{IF}}\left(t_{i}\right)\right)-\delta_{1, j}\left(t_{i}\right)-\lambda_{j} a_{1, j}^{s}-m_{\phi_{1, j}}^{s}\left(t_{i}\right)$ \\
$\tilde{d}_{r \neq 1, j>2}\left(t_{i}\right)$ & $d_{1 r, j}\left(t_{i}\right)-\left(d_{1 r, \mathrm{FF}}\left(t_{i}\right)+\mu_{j} d_{1 r, \mathrm{GF}}\left(t_{i}\right)\right)+m_{p_{1, j}}^{1}\left(t_{i}\right)$ \\
$\tilde{d}_{j>2}^{s}\left(t_{i}\right)$ & $d_{j, j}^{s}\left(t_{i}\right)-\left(d_{, \mathrm{IF}}^{s}\left(t_{i}\right)+\mu_{j} d_{, \mathrm{GF}}^{s}\left(t_{i}\right)\right)-\left(d_{1, j}\left(t_{i}\right)-\left(d_{1, \mathrm{FF}}\left(t_{i}\right)+\mu_{j} d_{1, \mathrm{GF}}\left(t_{i}\right)\right)\right)-m_{p_{1, j}}^{s}\left(t_{i}\right)$ \\
$\tilde{a}_{r \neq 1, j}^{s \neq 1}$ & $a_{1, j, j}^{s}-a_{1 r, j}^{1}$ \\
$S$-basis & $\mathrm{d} t_{1}\left(t_{i}\right), \delta_{1, j}\left(t_{i}\right), d_{1, j}\left(t_{i}\right), \bar{d}_{r \neq 1, j=1,2}\left(t_{i}\right), \bar{d}_{j=1,2}^{s}\left(t_{i}\right), a_{1, j}^{s}, a_{r, j}^{1}$ \\
\hline
\end{tabular}


filter (Odijk et al. 2017; Wang et al. 2017). The temporal behavior of the ZTDs is modeled by a random-walk process with a spectral density of $0.1 \mathrm{~mm} / \sqrt{s}$, and the ambiguities are assumed to be time-constant. The slant ionospheric delays and hardware biases are assumed to be unlinked. The double-differenced phase ambiguities $\tilde{a}$ are first estimated as float values in the Kalman filter. Using the LAMBDA method (Teunissen 1993, 1995) with the integer leastsquares (ILS) estimator, the real-valued ambiguities $\hat{\tilde{a}}$ are transformed and decorrelated, so that the ambiguity search ellipsoid is reformed to almost a spheroid, and fast and efficient integer least-squares estimation of the ambiguities can thus be enabled:

$\hat{z}=Z^{T} \hat{\tilde{a}}, \quad Q_{\hat{z} \hat{z}}=Z^{T} Q_{\hat{a} \hat{a}} Z$

where $\hat{z}$ and $Q_{\hat{z} \hat{z}}$ represent the transformed float ambiguities and their variance-covariance matrix, respectively. $Z$ denotes the transformation matrix that decorrelates the ambiguities $\hat{\tilde{a}}$, and $Q_{\hat{\hat{a}} \hat{a}}$ represents the variance-covariance matrix of the float ambiguities $\hat{\tilde{a}}$. After the decorrelation of the ambiguities, the ILS solutions are searched within a hyper-ellipsoidal space with iteratively reduced size (Chang et al. 2005; de Jonge and Tiberius 1996; Giorgi et al. 2008; Teunissen 1995, 2010).

The formal bootstrapping success rate (BSR), which lower bounds the success rate of the ILS estimator, is used as a measure for successful ambiguity resolution (Teunissen 1999):

$P\left(\check{z}_{\mathrm{LS}}=z\right) \geq P\left(\check{z}_{\mathrm{B}}=z\right) \geq \prod_{i=1}^{n}\left(2 \Phi\left(\frac{1}{2 \sigma_{\hat{z}_{i \mid l}}}\right)-1\right)$

with $\Phi(x)$ denoting the cumulative normal distribution function:

$\Phi(x)=\int_{-\infty}^{x} \frac{1}{\sqrt{2 \pi}} \exp \left(-\frac{u^{2}}{2}\right) \mathrm{d} u$

where $P\left(\check{z}_{\mathrm{LS}}=z\right)$ and $P\left(\check{z}_{\mathrm{B}}=z\right)$ represent the integer leastsquares success rate (LSR) and the integer BSR of the transformed ambiguities $z$, respectively. $n$ denotes the number of the transformed ambiguities. $\sigma_{\hat{z}_{i||}}$ with $I=i+1, \cdots, n$ stands for the conditional standard deviations of the transformed ambiguities $\hat{z}$, which are given by the diagonal matrix $\mathrm{D}$ after an $\mathrm{LDL}^{\mathrm{T}}$-decomposition of the matrix $Q_{\hat{\mathrm{z}} \hat{z}}$ (Teunissen 1999). $\exp (\cdot)$ denotes the natural exponential function.

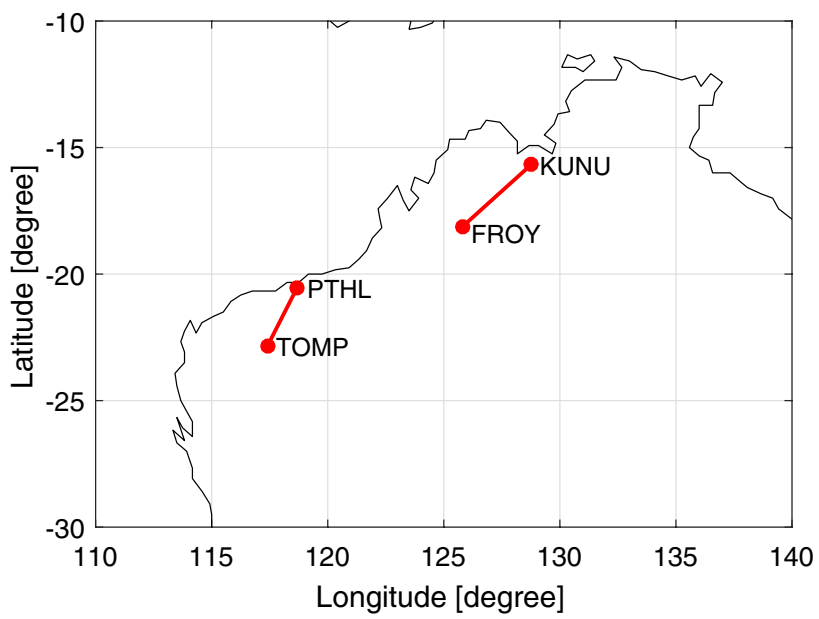

Fig. 1 West-Australia long baselines for Galileo processing. The length of the baselines FROY-KUNU and PTHL-TOMP amount to about 416 and $288 \mathrm{~km}$, respectively

Table 2 Details of the baselines used for the Galileo processing

\begin{tabular}{|c|c|c|c|c|}
\hline Baseline & $\begin{array}{l}\text { Baseline } \\
\text { length } \\
(\mathrm{km})\end{array}$ & Date & Time (GPST) & $\begin{array}{l}\text { No. of } \\
\text { satellites }\end{array}$ \\
\hline \multirow[t]{2}{*}{$\begin{array}{l}\text { FROY- } \\
\text { KUNU }\end{array}$} & \multirow[t]{2}{*}{416} & $\begin{array}{c}\text { April 19, } \\
2017\end{array}$ & $\begin{array}{r}18: 12: 20- \\
19: 29: 36\end{array}$ & 6 \\
\hline & & May 29, 2017 & $\begin{array}{r}15: 29: 57- \\
16: 46: 45\end{array}$ & 6 \\
\hline \multirow[t]{2}{*}{ PTHL-TOMP } & \multirow[t]{2}{*}{288} & $\begin{array}{l}\text { April 24, } \\
2017\end{array}$ & $\begin{array}{r}1: 26: 23- \\
3: 04: 28\end{array}$ & 6 \\
\hline & & June 2, 2017 & $\begin{array}{r}22: 44: 42- \\
23: 59: 59\end{array}$ & 6 \\
\hline
\end{tabular}

\section{Data selection}

Two long baselines in West-Australia are used for the processing as shown in Fig. 1. Galileo-only observations of $1 \mathrm{~Hz}$ are processed on two, three, four, and five frequencies. The elevation mask is set to be $10^{\circ}$. The length of the baselines, the time intervals used for the processing, and the number of the continuously tracked Galileo satellites are listed in Table 2. Septentrio receivers are used for both baselines. The Galileo frequency combinations used for the processing are listed in Table 3 with the frequency values given in RINEX (2015). The skyplots of the Galileo satellites for the stations FROY and PTHL are shown in Fig. 2. 
Table 3 Frequency combinations used for the processing

\begin{tabular}{ll}
\hline Number of frequencies & Signals \\
\hline 2 & E1, E5a \\
3 & E1, E5a, E5b \\
4 & E1, E5a, E5, E5b \\
5 & E1, E5a, E5, E5b, E6 \\
\hline
\end{tabular}
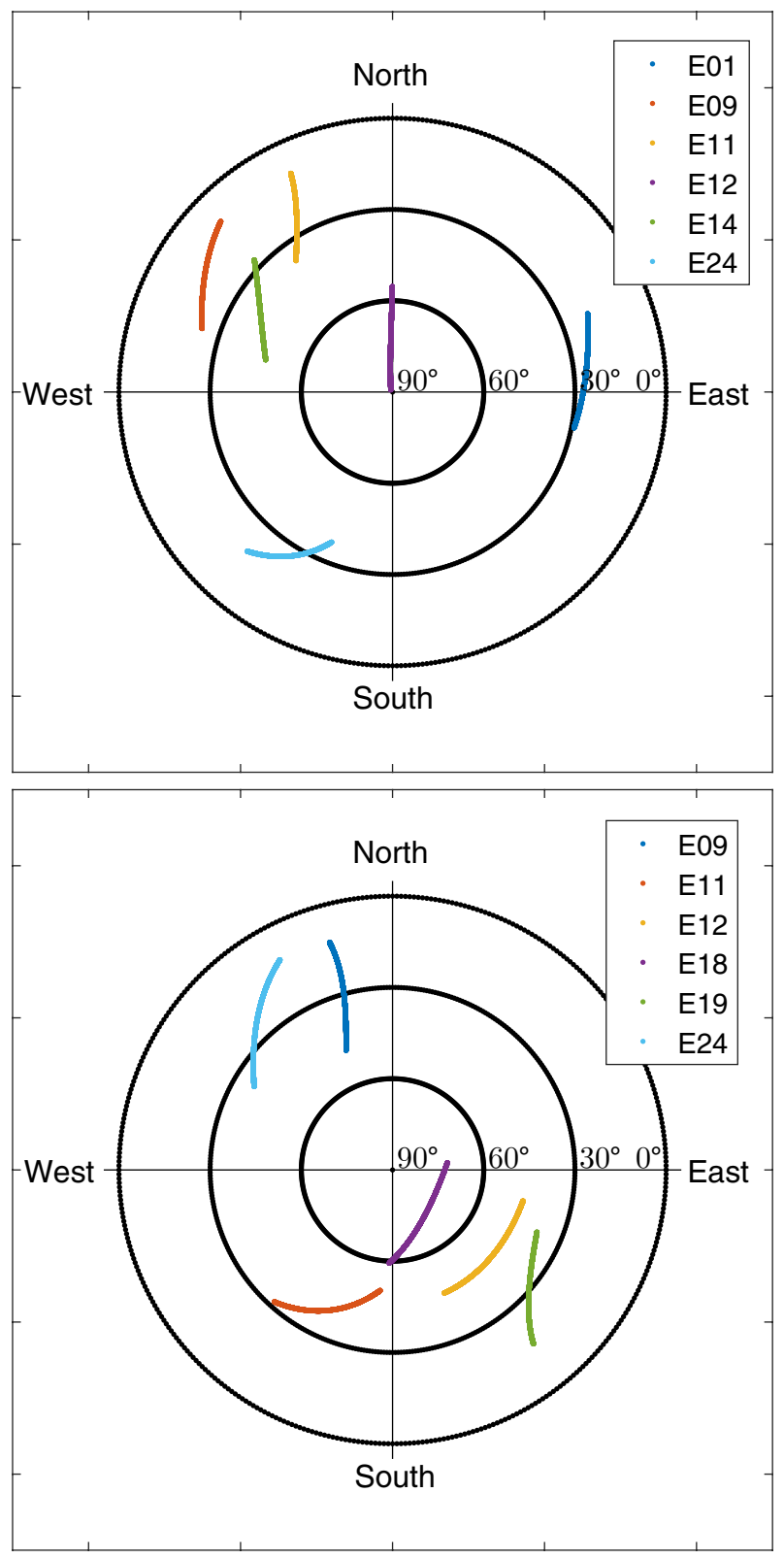

Fig. 2 Skyplots of the Galileo satellites. The top and the bottom panels show the skyplots for the station FROY in the first baseline on April 19, 2017 and the station PTHL in the second baseline on April 24, 2017 during the test time intervals (Table 2)

\section{Multipath mitigation}

As shown in (3) and (4), multipath effects are contained in the phase and code $\mathrm{O}-\mathrm{C}$ terms. To show an example of the multipath effects, we compute the double-differenced multipath combination $\mathrm{MP}_{1 r, j}^{1 s}$ (Leick et al. 2015) using the double-differenced phase $\left(\Delta \phi_{1 r, j}^{1 s}\right)$ and code O-C terms $\left(\Delta p_{1 r, j}^{1 s}\right)$ :

$$
\begin{aligned}
\mathrm{MP}_{1 r, j}^{1 s}= & \Delta p_{1 r, j}^{1 s}-\frac{\mu_{i}+\mu_{j}}{\mu_{i}-\mu_{j}}\left(\Delta \phi_{1 r, j}^{1 s}-\lambda_{j} \check{a}_{1 r, j}^{1 s}\right) \\
& +\frac{2 \mu_{j}}{\mu_{i}-\mu_{j}}\left(\Delta \phi_{1 r, i}^{1 s}-\lambda_{i} \check{a}_{1 r, i}^{1 s}\right) \\
= & m_{p_{1 r, j}}^{1 s}-\frac{\mu_{i}+\mu_{j}}{\mu_{i}-\mu_{j}} m_{\phi_{1 r, j}}^{1 s}+\frac{2 \mu_{j}}{\mu_{i}-\mu_{j}} m_{\phi_{1 r, i}}^{1 s}+\epsilon_{1 r, j}^{1 s}
\end{aligned}
$$

in which the fixed ambiguities $\check{a}_{1 r, j}^{1 s}$ and $\check{a}_{1 r, i}^{1 s}$ at the last epoch of the time interval are assumed to be the correctly resolved ambiguities, and $\epsilon_{1 r, j}^{1 s}$ represents the double-differenced multipath combination of the noise. As shown in (12), the multipath combination on frequency $j$ uses the double-differenced code $\mathrm{O}-\mathrm{C}$ terms on frequency $j$ and the double-differenced phase $\mathrm{O}-\mathrm{C}$ terms on frequency $j$ and another frequency $i$.

The multipath effects are assumed to repeat when the satellite constellation repeats for stationary networks. The Galileo IOV and FOC satellites have a repeat cycle of about 10 days (Zandbergen et al. 2004). According to ESA (2014) and ESA (2015), E18 and E14 were launched into incorrect orbits and reached their new target orbits in 2014 and 2015 , respectively. These two satellites repeat their ground tracks about every 20 days instead of 10 days as for the other Galileo satellites. For each baseline and each test day, we denote the tested time interval listed in Table 2 as $t$ with the $i$-th time point denoted as $t_{i}$. As the repeat times may vary within constellation and fluctuate for the same satellite (Agnew and Larson 2007), the repeat times were calculated for each satellite and each processing time point $t_{i}$. To correct multipath of satellite $s$ at the time point $t_{i}$, we used the MGEX precise orbits of the corresponding day and the day for multipath correction, i.e., May 9 and May 14, 2017 for baselines FROY-KUNU and PTHL-TOMP, respectively, to search for the time shift $\Delta T_{i}^{s}$, so that the following distance between satellite positions is minimized:

$D^{s}\left(t_{i}\right)=\frac{\sum_{r=1}^{2}\left|X^{s}\left(t_{i}-\beta_{r}^{s}\left(t_{i}\right)\right)-X^{s}\left(T_{i}^{s}-\beta_{r}^{s}\left(T_{i}^{s}\right)\right)\right|}{2}$

with

$T_{i}^{s}=t_{i}+\Delta T_{i}^{s}$ 

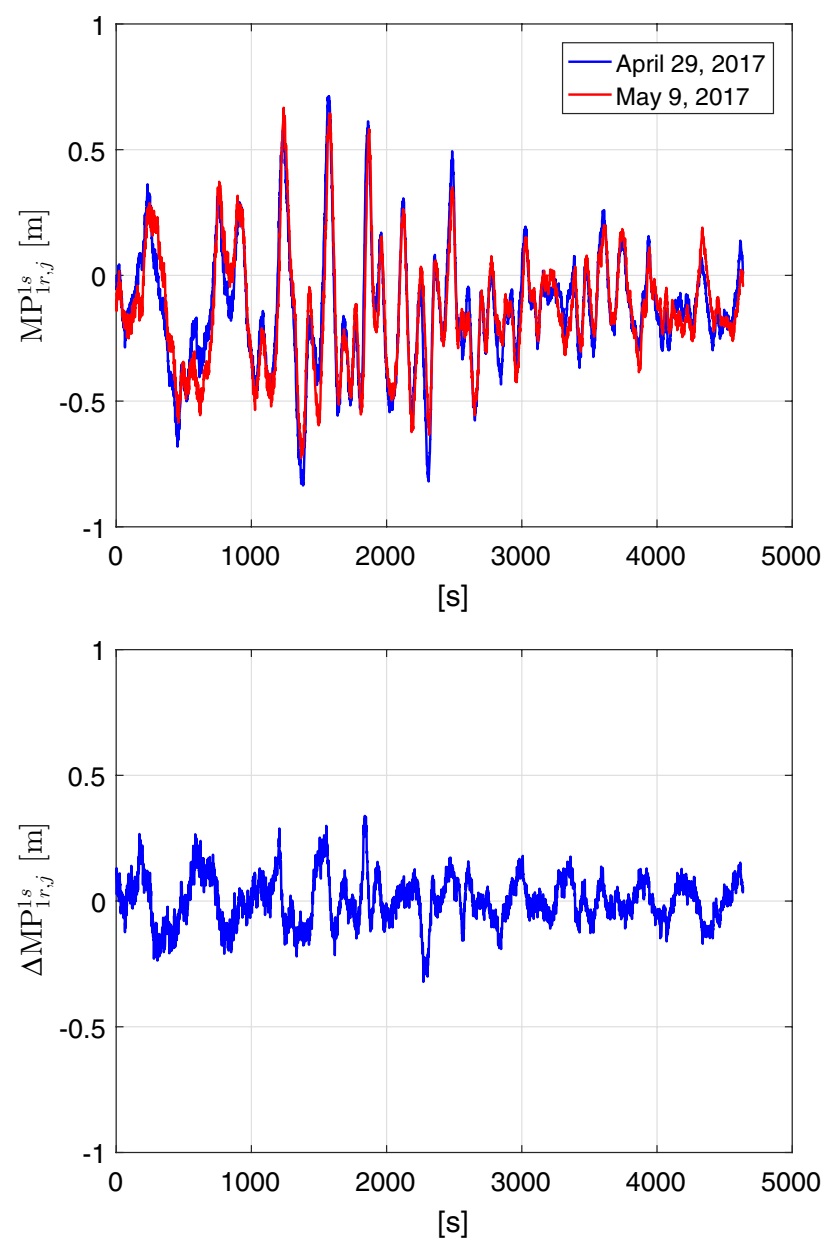

Fig. 3 (Top) Multipath combination on April 19, 2017 (blue) and at the shifted time points on May 9, 2017 (red), and (bottom) their differences. O-C terms on frequency E1 and E5 are used to compute the multipath combination on E5. The baseline FROY-KUNU and the satellites E12 and E11 are used for the plots

in which $\beta_{r}^{s}$ represents the signal running time from satellite $s$ to receiver $r$, and $X^{s}$ denotes the 3-dimensional orbit vector of satellite $s$. The search was performed on the basis of $1 \mathrm{~s}$, and $\Delta T_{i}^{s}$ denotes the searched time shift for satellite $s$ and $t_{i}$.

Figure 3 (top) shows the multipath combination $\mathrm{MP}_{1 r, j}^{1 s}$ on E5 for the baseline FROY-KUNU and the satellite pair E12 and E11 from 18:12:20 to 19:29:36 on April 19, 2017, plotted with the blue line, and at the shifted time points $T_{i}^{s}$ on May 9, 2017, plotted with the red line. To obtain the terms $\Delta \phi_{1 r, i}^{1 s}$ and $\check{a}_{1 r, i}^{1 s}$ in (12), the phase O-C terms and fixed ambiguities on $\mathrm{E} 1$ are also used for computing the multipath combination. We see that the multipath combination $\mathrm{MP}_{1 r, j}^{1 s}$ on April 19 has repeated behaviors on May 9, 2017. After forming differences between the blue and the red lines in Fig. 3 (top), as illustrated in the bottom panel of the figure, the multipath effects are reduced. The remaining systematic patterns are supposed to be caused by two reasons. First, by
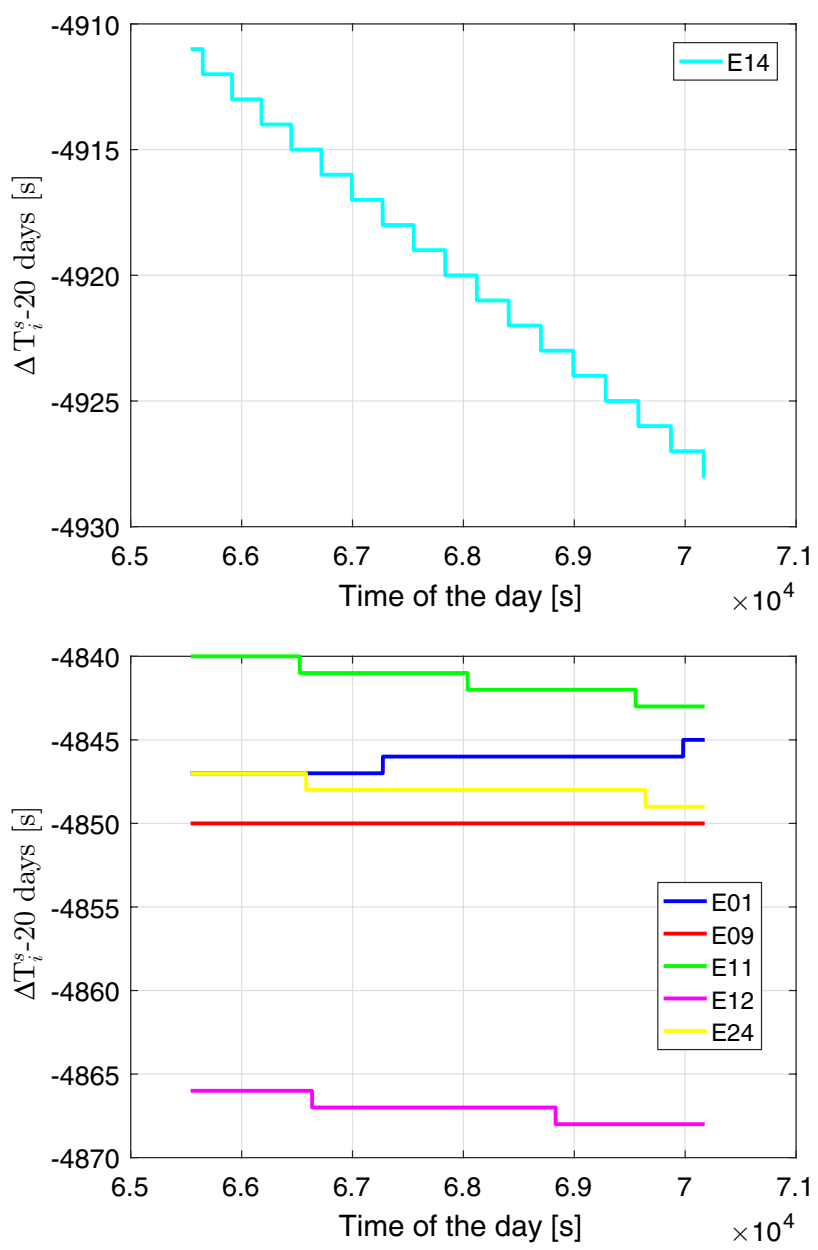

Fig. 4 Deviations of the time shifts for multipath mitigation from 20 days. The top panel shows the time shifts for E14 that was launched into incorrect orbits and recovered, and the bottom panel shows the other Galileo satellites for the baseline FROY-KUNU in the tested time interval on April 19, 2017 (Table 2)

computing the double-differenced multipath combination on May 9, 2017, the shifted time points $T_{i}^{s \neq 1}$ are used for both the reference satellite and the satellite $s$. This means that the multipath changes between $T_{i}^{1}$ and $T_{i}^{s}$ are not considered for the reference satellite. Second, the same satellite does not fly over exactly the same location after the searched time shift. The corresponding distances range from kilometers to tens of kilometers.

The multipath effects that are clearly observed in Fig. 3 may have significant influences on the ambiguity resolution performance. In the following two subsections, the procedure to mitigate multipath effects in the phase and code observations is explained in detail. In order to mitigate the multipath effects for the test time intervals, the Galileo measurements of the same station pairs, May 9 for the baseline FROY-KUNU and May 14, 2017 for the baseline PTHL-TOMP, are used with a time shift of about 20 days 
minus $80-82$ min. As an example, Fig. 4 shows the deviations of the time shifts $\Delta T_{i}^{s}$ from 20 days for all the six Galileo satellites and baseline FROY-KUNU in the tested time interval for April 19, 2017 (Table 2). We see that $\Delta T_{i}^{s}$ does not only differ for different satellites, but also changes during the tested time interval. Allowing a short initialization phase of the filter, the processing on the day used for multipath mitigation begins at $60 \mathrm{~s}$ earlier than the smallest $T_{i}^{s \neq 1}$ and ends at the largest $T_{i}^{s \neq 1}$. The entire processing interval on the day used for multipath mitigation is denoted as $T$. Since the multipath corrections are to be computed on doubledifference level at the shifted time point $T_{i}^{s \neq 1}$ for satellite $s \neq 1$, the shifted time points of the reference satellite $T_{i}^{1}$ are thus not considered when defining $T$.

\section{Residuals on the day used to compute multipath corrections}

For long baselines, the change of the between-receiver atmospheric delays in time hampers the mitigation of the multipath effects. Since the estimable tropospheric and ionospheric delays are estimated in the observation (3) and (4), we may utilize the double-differenced atmospheric estimates in $T$ to reduce the multipath effects in the test time intervals $t$ (Table 2).

The processing is first performed using the multipathuncorrected observations in $T$. The zenith-referenced a priori standard deviations for multipath-uncorrected Galileo observations given in Zaminpardaz and Teunissen (2017) for Septentrio receivers are used in this study. The exponential elevation weighting function (Euler and Goad 1991) is used for the processing:

$\sigma_{r, j}^{s^{2}}\left(t_{i}\right)=\sigma_{0, j}^{2}\left(a_{0}+a_{1} \exp \left(-\frac{e_{r}^{s}\left(t_{i}\right)}{e_{0}}\right)\right)^{2}$

where $\sigma_{0, j}$ and $\sigma_{r, j}^{s}\left(t_{i}\right)$ represent the a priori standard deviation of the observations in the zenith direction on frequency $j$ and the elevation-weighted standard deviation of the observation between receiver $r$ and satellite $s$ at the time point $t_{i}$ on frequency $j$, respectively. $e_{r}^{s}\left(t_{i}\right)$ stands for the elevation angle of the observations between receiver $r$ and satellite $s$ at the time point $t_{i}$ in degrees. The parameters $e_{0}, a_{0}$, and $a_{1}$ are pre-defined as $10^{\circ}, 1$, and 10 , respectively, as defined in Zaminpardaz and Teunissen (2017). As Odijk et al. (2014b) only found small cross-correlations between the E5/E5a/E5b signals, we assumed all the cross-correlations in this study to be zero.

The observation equations (3) and (4) during $T$, i.e., the time interval used to compute multipath corrections, can be formulated as follows:
$E(y)=A a+B b+[m]$

where $y$ stands for the vector of $\mathrm{O}-\mathrm{C}$ terms, and $a$ and $b$ represent the vector of the estimable ambiguities and other estimable parameters in Table 1, respectively. $A$ and $B$ denote the corresponding design matrices for $a$ and $b$, respectively. $m$ in $[\cdot]$ represents the multipath vector, which is not modeled. Considering the fact that no cycle slips are detected during $T$ with the help of the multipath combination (Leick et al. 2015), the resolved phase ambiguities for FAR at the last epoch of $T$ are taken as reference and used to estimate the other estimable parameters. After introducing the resolved ambiguities $\check{a}$ of the last epoch, the estimates of the observations $\check{y}$ can be formulated as follows:

$\check{y}=A \check{a}+B \check{b}$

where $\breve{b}$ represents the estimates of the other estimable parameters after introducing $\check{a}$.

Due to the robustness of integer ambiguity resolution ( $\mathrm{Li}$ et al. 2014), one may still obtain the correctly fixed ambiguities if the double-difference multipath is not too large. Assuming that the reference ambiguities at the last epoch of the time interval $T$ are correctly fixed with $\check{a}=a$, we can obtain the expectation of the residuals $\check{e}$ based on (16) and (17):

$E(\check{e})=E(y-\check{y})=B \cdot E(b-\breve{b})+[m]$

Based on (3), (4), and (18), the expectation of the doubledifferenced residuals of the phase observations $\check{e}_{\phi_{1 r, j}}^{1 s}$ and the code observations $\check{e}_{p_{1 r j}}^{1 s}$ after introducing the correctly fixed ambiguities of the last epoch can be formulated as follows:

$E\left(\check{e}_{\phi_{1 r, j}}^{1 s}\right)=g_{r}^{1 s} E\left(\Delta \check{\tau}_{r}\right)-g_{1}^{1 s} E\left(\Delta \check{\tau}_{1}\right)-\mu_{j} E\left(\Delta \check{\check{l}}_{1 r}^{1 s}\right)+\left[m_{\phi_{1 r, j}}^{1 s}\right]$

$E\left(\check{e}_{p_{1 r, j}}^{1 s}\right)=g_{r}^{1 s} E\left(\Delta \check{\tau}_{r}\right)-g_{1}^{1 s} E\left(\Delta \check{\tau}_{1}\right)+\mu_{j} E\left(\Delta \check{l}_{1 r}^{1 s}\right)+\left[m_{p_{1 r, j}}^{1 s}\right]$

with

$\Delta \check{\tau}_{r}=\tau_{r}-\check{\tau}_{r}$

$\Delta \check{l}_{1 r}^{1 s}=\tilde{l}_{1 r}^{1 s}-\check{l}_{1 r}^{1 s}$

where $\breve{\tau}_{r}$ and $\tilde{l}_{1 r}^{1 s}$ represent the estimated ZTD for receiver $r$ and the double-differenced ionospheric estimates at $T_{i}^{s}$, respectively, when introducing the fixed ambiguities of the last epoch. The double-differences are formed between both receivers and between the reference satellite and the satellite $s \neq 1$ at the time point $T_{i}^{s \neq 1}$. 


\section{Multipath corrections applied to current data}

Assuming that the satellite configuration and the design matrices $A$ and $B$ in the time interval $T$ given in (16) repeat in the current test time interval $t$, the observation equations of the current test time interval $t$ can be formulated as follows:

$E\left(y_{0}\right)=A a_{0}+B b_{0}+\left[m_{0}\right]$

where $y_{0}, a_{0}, b_{0}$, and $m_{0}$ represent the vector of the O-C terms, the estimable ambiguities, the other estimable parameters (Table 1), and the multipath during the test time interval $t$, respectively. After subtracting the residuals $\check{e}$ in the time interval $T$ from the $y_{0}$ in the current test time interval $t$, based on (18) and (23), the corrected observation equations can be formulated as follows:

$E\left(y_{0}-\check{e}\right)=A a_{0}+B\left(b_{0}-E(b-\check{b})\right)+[\Delta m]$

with

$\Delta m=m_{0}-m$

Under the assumption that the satellite configuration repeats in $T$ and $t$, the multipath is assumed to be removed, i.e., $\Delta m=0$.

In practice, however, the time shifts $\Delta T_{i}^{s}$ of different Galileo satellites are different. In order to correct the multipath at the time point $t_{i}$ in the current test time interval, the residuals of different time points $T_{i}^{s}$ are used for different satellites. As stated in the previous subsection, double-differences of the residuals are generated between the reference satellite and satellite $s$ at the time point $T_{i}^{s \neq 1}$. The corrected $\mathrm{O}-\mathrm{C}$ terms of the phase and the code observations at $t_{i}$ during current processing time interval after subtracting the double-differenced residuals $\check{e}_{\phi_{1 r, j}^{1 s}}^{1 s}$ and $\check{e}_{p_{1 r, j}}^{1 s}$ at $T_{i}^{s \neq 1}$ can be reformulated as follows:

$$
\begin{aligned}
E\left(\Delta \phi_{r, j}^{s}\left(t_{i}\right)-\check{e}_{\phi_{1, j}}^{1 s}\left(T_{i}^{s}\right)\right)= & g_{r}^{s} \tilde{\tilde{\tau}}_{r}+\mathrm{d} \tilde{\tilde{t}}_{r}-\mathrm{d} \tilde{\tilde{t}}^{s}-\mu_{j} \tilde{\tilde{l}}_{r}^{s}+\tilde{\delta}_{r, j}-\tilde{\delta}_{j}^{s} \\
& +\lambda_{j} \tilde{a}_{r, j}^{s}+\left[\Delta m_{\phi_{1, j}}^{1 s}\right]
\end{aligned}
$$

$$
\begin{aligned}
E\left(\Delta p_{r, j}^{s}\left(t_{i}\right)-\check{e}_{p_{1, j}}^{1 s}\left(T_{i}^{s}\right)\right)= & g_{r}^{s} \tilde{\tilde{\tau}}_{r}+\mathrm{d} \tilde{t}_{r}-\mathrm{d} \tilde{t}^{s} \\
& +\mu_{j} \tilde{\tilde{l}}_{r}^{s}+\tilde{d}_{r, j}-\tilde{d}_{j}^{s}+\left[\Delta m_{p_{1, j}}^{1 s}\right]
\end{aligned}
$$

where the estimable parameters and the ZTD mapping function $g_{r}^{s}$ refer to the time point $t_{i}$, and the new estimable parameters $\tilde{\tilde{\tau}}_{r}$, $\tilde{\tilde{t}}_{r}, \mathrm{~d} \tilde{\tilde{t}}^{s}$, and $\tilde{\tilde{l}}_{r}^{s}$ at the time point $t_{i}$ are listed in Table 4 with detailed explanation given in "Appendix A." Due to the different time shifts for different Galileo satellites, the multipath effects of the reference satellite cannot be completely removed, and the terms $g_{r}^{1 s} E\left(\Delta \check{\tau}_{r}\right)$ and $g_{1}^{1 s} E\left(\Delta \check{\tau}_{1}\right)$ in (19) and (20) cannot be fully absorbed by the new estimable parameters in Table 4 . These effects are contained in $\Delta m_{\phi_{1 r, j}}^{1 s}$ and $\Delta m_{p_{1, j}}^{1 s}$ in (26) and (27) with the evaluation given in "Appendix A." This means that multipath can be mitigated, but residual biases may still be present due to the different time shifts of Galileo satellites. As remarked before, the satellite does not fly over exactly the same position after the searched time shift, i.e., with distances within several tens of kilometers. This would also lead to incomplete removal of the multipath effects.

Due to the influences of the multipath effects during $T$, the expectations of $\Delta \check{\tau}_{r}$ and $\Delta \check{\tilde{l}}_{1 r}^{l s}$ in (21) and (22) do not equal to zero. Compared to the case without multipath mitigation, the new estimable parameters in Table 4 during the current processing time interval $t$ are thus still influenced by the multipath effects during $T$, which are generally within millimeters at the zenith direction for Galileo phase signals (Cai et al. 2016; Zaminpardaz and Teunissen 2017). However, since the double-differenced multipath during $t$ is significantly mitigated by subtracting the residuals during $T$, the ambiguity resolution during $t$ is accelerated. The results will be discussed in the next section.

Instead of using the observation equations in (3) and (4), the corrected observation (26) and (27) are used for the processing in Kalman filter. The variance matrix of the corrected observations is approximated to a diagonal matrix with the diagonal elements computed using the exponential elevation weighting function given in (15). The undifferenced zenith-referenced a priori standard deviations of the multipath-corrected Galileo observations reported in Zaminpardaz and Teunissen (2017) for Septentrio receivers are

Table 4 New estimable parameters in (26) and (27) at $t_{i}$ during the current processing time interval $t$ after subtracting double-differenced residuals at $T_{i}^{s}$. The ionospheric delays and the hardware biases are estimated as unlinked parameters, and the ZTDs are linked in time

\begin{tabular}{ll}
\hline Parameter & Interpretation \\
\hline$\tilde{\tau}_{r}\left(t_{i}\right)$ & $\tau_{r}\left(t_{i}\right)-E\left(\Delta \check{t}_{r}\left(T_{i}^{2}\right)\right)$ \\
$\mathrm{d} \tilde{t}_{r \neq 1}\left(t_{i}\right)$ & $\mathrm{d} t_{1 r}\left(t_{i}\right)+d_{1 r, I F}\left(t_{i}\right)+g_{r}^{1}\left(T_{i}^{1}\right) E\left(\Delta \check{\tau}_{r}\left(T_{i}^{2}\right)\right)-g_{1}^{1}\left(T_{i}^{1}\right) E\left(\Delta \check{\tau}_{1}\left(T_{i}^{2}\right)\right)$ \\
$\tilde{\mathrm{d}}_{\tilde{\tau}}^{s}\left(t_{i}\right)$ & $\mathrm{d} s\left(t_{i}\right)+d_{\mathrm{IF}}^{s}\left(t_{i}\right)-\left(\mathrm{d} t_{1}\left(t_{i}\right)+d_{1, \mathrm{IF}}\left(t_{i}\right)\right)-g_{1}^{s}\left(T_{i}^{s}\right) E\left(\Delta \check{\tau}_{1}\left(T_{i}^{2}\right)\right)$ \\
$\tilde{\tilde{t}}_{r}^{s}\left(t_{i}\right)$ & $t_{r}^{s}\left(t_{i}\right)+d_{r, \mathrm{GF}}\left(t_{i}\right)-d_{\mathrm{GF}}^{s}\left(t_{i}\right)-E\left(\Delta \check{l}_{1 r}^{s}\left(T_{i}^{s}\right)\right)$ \\
\hline
\end{tabular}


used in this study. After subtraction of the residuals at $T_{i}^{S}$, the undifferenced a priori standard deviations are multiplied with a factor of $\sqrt{2}$.

\section{Analysis of the results}

Formal and empirical analysis are performed using the data from both baselines and all tested time intervals listed in Table 2. A time window of $15 \mathrm{~min}$ is defined for each round of processing and is shifted by $30 \mathrm{~s}$ before restarting the next round of processing. The empirical results using multipathmitigated and -uncorrected observations are compared with the formal results in the end of the section.

\section{Formal analysis}

Based on the model described before, the mean formal BSR at the time point $t_{i}$, denoted as $\bar{P}_{\mathrm{F}}\left(t_{i}\right)$, can be calculated using both baselines and test intervals in Table 2:

$\bar{P}_{\mathrm{F}}\left(t_{i}\right)=\frac{\sum_{q=1}^{l} \sum_{k=1}^{h(q)} \sum_{p=1}^{w(q, k)} P_{\mathrm{BSR}}^{q, k, p}\left(t_{i}\right)}{\sum_{q=1}^{l} \sum_{k=1}^{h(q)} w(q, k)}$

where $l, h(q)$, and $w(q, k)$ denote the number of baselines, the number of test intervals for baseline $q$ and the number of time windows for baseline $q$ and test interval $k$, respectively. $P_{\mathrm{BSR}}^{q, k, p}$ refers to the formal BSR for baseline $q$ and time window $p$ in the test interval $k$.

Figure 5 shows the mean formal BSRs $\bar{P}_{\mathrm{F}}$ for different frequency combinations using all data sets. The horizontal black-dashed lines mark the $\bar{P}_{\mathrm{F}}$ of 90 and $99 \%$. From Fig. 5, we see that the $\bar{P}_{\mathrm{F}}$ increase with the increasing number of frequencies. The mean formal BSR $\bar{P}_{\mathrm{F}}$ at $t_{i}$ of 5,10 , and

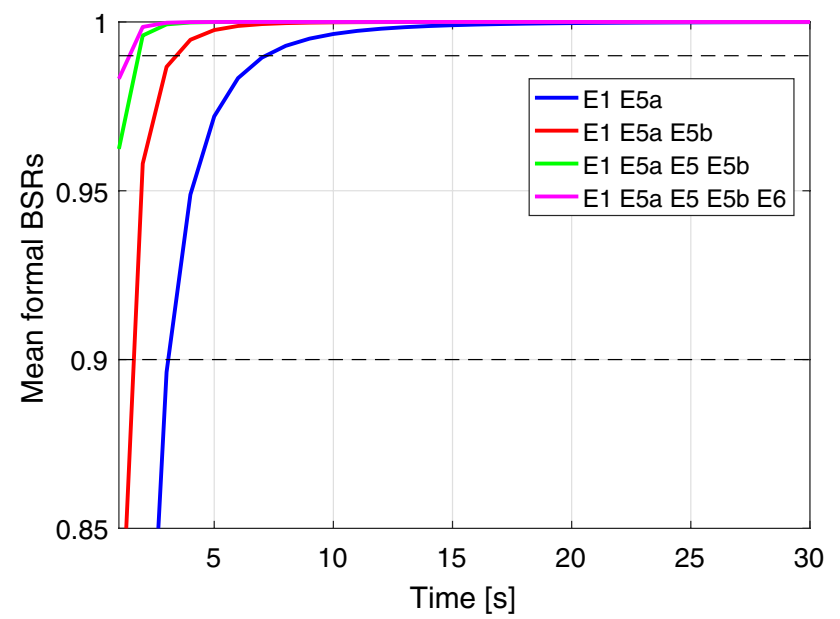

Fig. 5 Mean formal bootstrapping success rates (BSRs) $\bar{P}_{\mathrm{F}}$ given in (28). $1 \mathrm{~Hz}$ data for both baselines and all time windows in the tested time intervals (Table 2) are used for the plot
Table 5 Mean formal bootstrapping success rates (BSRs) $\bar{P}_{\mathrm{F}}$ in percent given in (28) at 5, 10, and $20 \mathrm{~s}$ of the processing

\begin{tabular}{lrrr}
\hline Frequencies & \multicolumn{1}{l}{$5 \mathrm{~s}$} & \multicolumn{1}{l}{$10 \mathrm{~s}$} & \multicolumn{1}{c}{$20 \mathrm{~s}$} \\
\hline $\mathrm{E} 1+\mathrm{E} 5 \mathrm{a}$ & 97.20 & 99.64 & 99.97 \\
$\mathrm{E} 1+\mathrm{E} 5 \mathrm{a}+\mathrm{E} 5 \mathrm{~b}$ & 99.76 & 99.99 & $>99.99$ \\
$\mathrm{E} 1+\mathrm{E} 5 \mathrm{a}+\mathrm{E} 5+\mathrm{E} 5 \mathrm{~b}$ & $>99.99$ & $>99.99$ & $>99.99$ \\
$\mathrm{E} 1+\mathrm{E} 5 \mathrm{a}+\mathrm{E} 5+\mathrm{E} 5 \mathrm{~b}+\mathrm{E} 6$ & $>99.99$ & $>99.99$ & $>99.99$ \\
\hline
\end{tabular}

$20 \mathrm{~s}$ are listed in Table 5 for different frequency combinations. Using three or more frequencies, the $\bar{P}_{\mathrm{F}}$ are larger than $99.7 \%$ after $5 \mathrm{~s}$. After $20 \mathrm{~s}$, all frequency combinations deliver $\bar{P}_{\mathrm{F}}$ larger than $99.9 \%$. We remark that the formal BSRs are computed based on the assumption that the multipath effects are completely removed from the observations.

\section{Empirical analysis}

As stated before, in practice, the multipath effects cannot be completely removed. Using both the multipath-mitigated and -uncorrected Galileo observations for both baselines and all time windows, the mean empirical success rate $\bar{P}_{\mathrm{E}}$ can be calculated for each time point $t_{i}$ :

$\bar{P}_{\mathrm{E}}\left(t_{i}\right)=\frac{\sum_{q=1}^{l} \sum_{k=1}^{h(q)} \sum_{p=1}^{w(q, k)} P_{\mathrm{E}}^{q, k, p}\left(t_{i}\right)}{\sum_{q=1}^{l} \sum_{k=1}^{h(q)} w(q, k)}$

where the empirical success rate $P_{\mathrm{E}}^{q, k, p}\left(t_{i}\right)$ is defined as the ratio of the number of the correctly fixed ambiguities $N_{\mathrm{C}}^{q, k, p}$ to the number of all ambiguities $N^{q, k, p}$ for baseline $q$ in the test window $p$ of the test interval $k$ at the time point $t_{i}$ :

$P_{\mathrm{E}}^{q, k, p}\left(t_{i}\right)=\frac{N_{\mathrm{C}}^{q, k, p}\left(t_{i}\right)}{N^{q, k, p}\left(t_{i}\right)}$

We remark that $N_{\mathrm{C}}^{q, k, p}$ and $N^{q, k, p}$ in (30) refer to the transformed ambiguities. Here, we use the fixed ambiguities at the last epoch of the entire test time interval as reference values to calculate the correctly fixed transformed ambiguities at each epoch.

Figure 6 shows the mean empirical success rates (SRs) $\bar{P}_{\mathrm{E}}$ using both baselines and all time windows in the tested time intervals. In the top panel, the multipath effects are mitigated as described in the last section. In contrast to the multipath-mitigated case, we also compute the $\bar{P}_{\mathrm{E}}$ without correcting the multipath effects using the zenith-referenced a priori standard deviations of the multipath-uncorrected observations given in Zaminpardaz and Teunissen (2017) for Septentrio receivers.

From Fig. 6, we see that the multipath mitigation has increased the mean empirical SRs compared to the case 

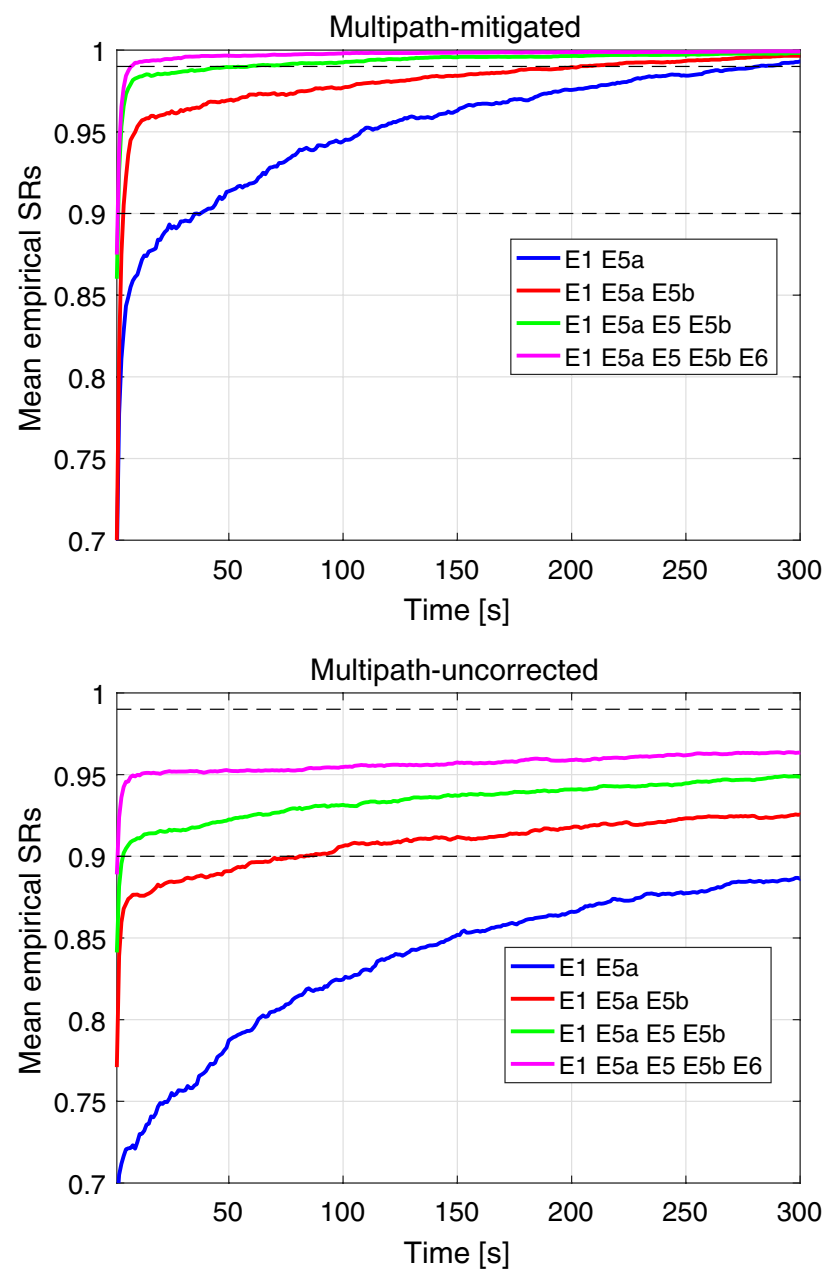

Fig. 6 Mean empirical success rates (SRs) $\bar{P}_{\mathrm{E}}$ given in (28). The multipath-mitigated and -uncorrected observations are used for the plots in the top and bottom panels. $1 \mathrm{~Hz}$ data of both baselines and all time windows in the tested time intervals (Table 2) are used for the processing

using multipath-uncorrected observations. In both cases, a higher number of frequencies is helpful to increase the SRs of ambiguity fixing. The mean empirical SRs $\bar{P}_{\mathrm{E}}$ at 5,10 , $20 \mathrm{~s}, 1$, and 2 min are listed in Table 6 using multipath-mitigated and -uncorrected observations, respectively. After the multipath mitigation, the $\bar{P}_{\mathrm{E}}$ is higher than 95,98 , and $99 \%$ at $10 \mathrm{~s}$ using three, four, and five frequencies, respectively, while the values remain at around 88,91, and 95\% using multipath-uncorrected observations, respectively.

Comparing the mean formal BSRs in the last subsection and the mean empirical SRs using multipath-mitigated and -uncorrected observations, we observe that the differences after the multipath mitigation are reduced. Table 7 lists the differences between the mean formal BSRs and the mean empirical SRs with and without multipath mitigation. At $t_{i}$ of $10 \mathrm{~s}$, the differences in mean SRs amount to around 12,9 , and $5 \%$ using multipath-uncorrected observations on
Table 6 Mean empirical success rates (SRs) $\bar{P}_{\mathrm{E}}$ in percent given in (28) at 5, 10, $20 \mathrm{~s}, 1$, and 2 min of the processing using multipathmitigated and multipath-uncorrected observations

\begin{tabular}{llllll}
\hline Frequencies & $5 \mathrm{~s}$ & $10 \mathrm{~s}$ & $20 \mathrm{~s}$ & $1 \mathrm{~min}$ & $2 \mathrm{~min}$ \\
\hline Multipath-mitigated & & & & & \\
E1+E5a & 84.34 & 86.26 & 88.38 & 91.84 & 95.51 \\
E1+E5a+E5b & 92.19 & 95.32 & 96.00 & 97.28 & 98.09 \\
E1+E5a+E5+E5b & 97.31 & 98.31 & 98.52 & 99.03 & 99.45 \\
E1+E5a+E5+E5b+E6 & 98.43 & 99.23 & 99.37 & 99.66 & 99.82 \\
Multipath-uncorrected & & & & & \\
E1+E5a & 72.07 & 72.51 & 74.90 & 79.37 & 83.78 \\
E1+E5a+E5b & 87.09 & 87.66 & 88.16 & 89.63 & 90.79 \\
E1+E5a+E5+E5b & 90.47 & 91.11 & 91.55 & 92.50 & 93.32 \\
E1+E5a+E5+E5b+E6 & 94.56 & 94.99 & 95.05 & 95.24 & 95.58 \\
\hline
\end{tabular}

three, four, and five frequencies, respectively, while they are reduced to below 5,2 , and $1 \%$ using multipath-mitigated observations.

\section{Conclusions}

For long-baseline ambiguity resolution, the ionospheric delays that cannot be removed by generating between-station differences hampers rapid ambiguity resolution. The firstorder terms of the ionospheric delays can either be eliminated by forming linear combinations using multi-frequency data, or estimated as float values using uncombined phase and code observations. The latter strategy has the advantage that it keeps all information in the observation equations and is easy to be expanded to any number of frequencies. The dual-frequency case is, however, found to be too weak to use such a model. With the increasing number of the frequencies in current GNSS, it is now possible to exploit the benefits of

Table 7 Differences between the mean formal and empirical success rates (SRs) in percent using multipath-mitigated and multipath-uncorrected observations

\begin{tabular}{lrrrrr}
\hline Frequencies & \multicolumn{1}{c}{$5 \mathrm{~s}$} & $10 \mathrm{~s}$ & $20 \mathrm{~s}$ & $1 \mathrm{~min}$ & $2 \mathrm{~min}$ \\
\hline Multipath-mitigated & & & & & \\
E1+E5a & 12.86 & 13.39 & 11.59 & 8.16 & 4.49 \\
E1+E5a+E5b & 7.57 & 4.67 & 4.00 & 2.72 & 1.91 \\
E1+E5a+E5+E5b & 2.69 & 1.69 & 1.48 & 0.97 & 0.55 \\
E1+E5a+E5+E5b+E6 & 1.57 & 0.77 & 0.63 & 0.34 & 0.18 \\
Multipath-uncorrected & & & & & \\
E1+E5a & 25.14 & 27.13 & 25.07 & 20.63 & 16.22 \\
E1+E5a+E5b & 12.67 & 12.33 & 11.84 & 10.37 & 9.21 \\
E1+E5a+E5+E5b & 9.52 & 8.89 & 8.45 & 7.50 & 6.68 \\
E1+E5a+E5+E5b+E6 & 5.44 & 5.01 & 4.95 & 4.76 & 4.42 \\
\hline
\end{tabular}


real multi-frequency data in long-baseline ambiguity resolution using uncombined phase and code observations.

The current Galileo system is providing signals on five frequencies on 15 operational IOV and FOC satellites by the end of September 2017. In this study, real Galileo data on all five frequencies for two baselines of several hundreds of kilometers in Australia are used for the processing. Using both multipath-mitigated and -uncorrected phase and the code observations, we studied the formal and the empirical ambiguity success rates for long baselines with different frequency combinations. We observed that the multipath mitigation procedure is important for improving the empirical ambiguity success rates compared to the cases using multipath-uncorrected observations. A higher number of frequencies are also found to be essential for achieving high success rates within short time. Using $1 \mathrm{~Hz}$ data after multipath mitigation, the mean empirical success rates are found to be above 95,98 , and $99 \%$ after $10 \mathrm{~s}$ using three, four, and five frequencies, respectively.

Acknowledgements We would like to thank the International GNSS Service (IGS) and Geoscience Australia for providing the MGEX orbit products, the observation data and the station coordinates. The MGEX orbit products were obtained through the online archives of the Crustal Dynamics Data Information System (CDDIS), NASA Goddard Space Flight Center, Greenbelt, MD, USA. ftp://cddis.gsfc.nasa.gov/gnss/ products/mgex/. We are also grateful for the contributions of our colleagues in the GNSS Research Centre, Curtin University, on the development of the Curtin PPP-RTK Software. PJG Teunissen is recipient of an Australian Research Council (ARC) Federation Fellowship (project number FF0883188)

Open Access This article is distributed under the terms of the Creative Commons Attribution 4.0 International License (http://creativeco mmons.org/licenses/by/4.0/), which permits unrestricted use, distribution, and reproduction in any medium, provided you give appropriate credit to the original author(s) and the source, provide a link to the Creative Commons license, and indicate if changes were made.

\section{Appendix A: Unmodeled effects}

Based on (19) and (20), the expectation of the double-differenced phase and code residuals at $T_{i}^{s \neq 1}$ can be formulated as follows:

$$
\begin{aligned}
& E\left(\check{e}_{\phi_{1, j}}^{1 s}\left(T_{i}^{s}\right)\right)=E\left(D_{1 r}^{1 s}\left(T_{i}^{s}\right)\right)-\mu_{j} E\left(\Delta \check{\tilde{l}}_{1 r}^{1 s}\left(T_{i}^{s}\right)\right)+\left[\begin{array}{r}
\left.m_{\phi_{1, j}}^{1 s}\left(T_{i}^{s}\right)\right] \\
(31)
\end{array}\right. \\
& E\left(\check{e}_{p_{1 r, j}}^{1 s}\left(T_{i}^{s}\right)\right)=E\left(D_{1 r}^{1 s}\left(T_{i}^{s}\right)\right)+\mu_{j} E\left(\Delta \check{\bar{l}}_{1 r}^{1 s}\left(T_{i}^{s}\right)\right)+\left[m_{p_{1 r, j}}^{1 s}\left(T_{i}^{s}\right)\right]
\end{aligned}
$$

with

$$
\begin{aligned}
D_{1 r}^{1 s}\left(T_{i}^{s}\right)= & g_{r}^{1 s}\left(T_{i}^{s}\right) \Delta \check{\tau}_{r}\left(T_{i}^{s}\right)-g_{1}^{1 s}\left(T_{i}^{s}\right) \Delta \check{\tau}_{1}\left(T_{i}^{s}\right) \\
= & \left(g_{r}^{s}\left(T_{i}^{s}\right) \Delta \check{\tau}_{r}\left(T_{i}^{s}\right)-g_{r}^{1}\left(T_{i}^{s}\right) \Delta \check{\tau}_{r}\left(T_{i}^{s}\right)\right) \\
& -\left(g_{1}^{s}\left(T_{i}^{s}\right) \Delta \check{\tau}_{1}\left(T_{i}^{s}\right)-g_{1}^{1}\left(T_{i}^{s}\right) \Delta \check{\tau}_{1}\left(T_{i}^{s}\right)\right)
\end{aligned}
$$

In order to let the term $D_{1 r}^{1 s}\left(T_{i}^{s}\right)$ possibly be absorbed by the estimable parameters during $t$, the ZTD mapping function $g_{r}^{1}\left(T_{i}^{s}\right)$ and $g_{1}^{1}\left(T_{i}^{s}\right)$ are replaced by $g_{r}^{1}\left(T_{i}^{1}\right)$ and $g_{1}^{1}\left(T_{i}^{1}\right)$, and the terms $\Delta \check{\tau}_{r}\left(T_{i}^{s}\right)$ and $\Delta \check{\tau}_{1}\left(T_{i}^{s}\right)$ (with $\left.s \neq 1\right)$ are replaced by $\Delta \check{\tau}_{r}\left(T_{i}^{2}\right)$ and $\Delta \check{\tau}_{1}\left(T_{i}^{2}\right)$, which refer to the same time point $T_{i}^{2}$ for all satellites pairs:

$$
\begin{aligned}
D_{1 r}^{1 s}\left(T_{i}^{s}\right)= & \left(g_{r}^{s}\left(T_{i}^{s}\right) \Delta \check{\tau}_{r}\left(T_{i}^{2}\right)+g_{r}^{s}\left(T_{i}^{s}\right) \Delta \check{\tau}_{r}\left(T_{i}^{2 s}\right)\right) \\
& -\left(g_{r}^{1}\left(T_{i}^{1}\right) \Delta \check{\tau}_{r}\left(T_{i}^{2}\right)+g_{r}^{1}\left(T_{i}^{1 s}\right) \Delta \check{\tau}_{r}\left(T_{i}^{2}\right)\right. \\
& \left.+g_{r}^{1}\left(T_{i}^{s}\right) \Delta \check{\tau}_{r}\left(T_{i}^{2 s}\right)\right) \\
& -\left(g_{1}^{s}\left(T_{i}^{s}\right) \Delta \check{\tau}_{1}\left(T_{i}^{2}\right)+g_{1}^{s}\left(T_{i}^{s}\right) \Delta \check{\tau}_{1}\left(T_{i}^{2 s}\right)\right) \\
& +\left(g_{1}^{1}\left(T_{i}^{1}\right) \Delta \check{\tau}_{1}\left(T_{i}^{2}\right)+g_{1}^{1}\left(T_{i}^{1 s}\right) \Delta \check{\tau}_{1}\left(T_{i}^{2}\right)\right. \\
& \left.+g_{1}^{1}\left(T_{i}^{s}\right) \Delta \check{\tau}_{1}\left(T_{i}^{2 s}\right)\right) \\
= & D 0_{1 r}^{1 s}\left(T_{i}^{s}\right)-\Delta H_{r \neq 1}^{s \neq 1}\left(T_{i}^{s \neq 1}\right)-\Delta G_{r \neq 1}^{s \neq 1,2}\left(T_{i}^{s \neq 1,2}\right)
\end{aligned}
$$

where the first, third, sixth, and eighth parameters are summarized by the term $D 0_{1 r}^{1 s}\left(T_{i}^{s}\right)$. The second, fifth, seventh, and tenth parameters are included in the bias term $\Delta G_{r \neq 1}^{s \neq 1,2}\left(T_{i}^{s \neq 1,2}\right)$, and the fourth and ninth parameters are contained in the bias term $\Delta H_{r \neq 1}^{s \neq 1}\left(T_{i}^{s \neq 1}\right)$ :

$\Delta G_{r \neq 1}^{s \neq 1,2}\left(T_{i}^{s \neq 1,2}\right)=g_{1}^{1 s}\left(T_{i}^{s}\right) \Delta \check{\tau}_{1}\left(T_{i}^{2 s}\right)-g_{r}^{1 s}\left(T_{i}^{s}\right) \Delta \check{\tau}_{r}\left(T_{i}^{2 s}\right)$

$\Delta H_{r \neq 1}^{s \neq 1}\left(T_{i}^{s \neq 1}\right)=g_{r}^{1}\left(T_{i}^{1 s}\right) \Delta \check{\tau}_{r}\left(T_{i}^{2}\right)-g_{1}^{1}\left(T_{i}^{1 s}\right) \Delta \check{\tau}_{1}\left(T_{i}^{2}\right)$

The formulation $(\cdot)\left(T_{i}^{p s}\right)$ in (34), (35), and (36) represents:

$(\cdot)\left(T_{i}^{p s}\right)=(\cdot)\left(T_{i}^{s}\right)-(\cdot)\left(T_{i}^{p}\right)$

After subtracting the expected double-differenced residuals at $T_{i}^{s \neq 1}$ from the expected O-C terms of the phase and the code observations at $t_{i}$, as performed in (26) and (27), the expectations of the parameters included in $D 0_{1 r}^{1 s}\left(T_{i}^{s}\right)$ (34) and the parameter $\Delta \check{l}_{1 r}^{l s}\left(T_{i}^{s}\right)$ in (31) and (32) are absorbed by the new estimable parameters in Table 4 . The terms $\Delta m_{\phi_{1 r, j}}^{1 s}$ and $\Delta m_{p_{1 r, j}}^{1 s}$ in (26) and (27) are thus formulated as follows:

$$
\begin{aligned}
\Delta m_{\phi_{1 r, j}}^{1 s}\left(t_{i}\right)= & E\left(\Delta G_{r \neq 1}^{s \neq 1,2}\left(T_{i}^{s \neq 1,2}\right)\right)+E\left(\Delta H_{r \neq 1}^{s \neq 1}\left(T_{i}^{s \neq 1}\right)\right) \\
& +m_{\phi_{1, j}}^{1 s}\left(t_{i}\right)-m_{\phi_{1, j}}^{1 s}\left(T_{i}^{s}\right) \\
\Delta m_{p_{1, j}}^{1 s}\left(t_{i}\right)= & E\left(\Delta G_{r \neq 1}^{s \neq 1,2}\left(T_{i}^{s \neq 1,2}\right)\right)+E\left(\Delta H_{r \neq 1}^{s \neq 1}\left(T_{i}^{s \neq 1}\right)\right) \\
& +m_{p_{1 r, j}}^{1 s}\left(t_{i}\right)-m_{p_{1 r, j}}^{1 s}\left(T_{i}^{s}\right)
\end{aligned}
$$

We assume that the multipath effects of receiver $r$ and satellite $s$ at $t_{i}$ repeat at $T_{i}^{s}$, the multipath differences in (38) and (39) can then be reformulated as follows: 


$$
\begin{aligned}
& m_{\phi_{1 r, j}}^{1 s}\left(t_{i}\right)-m_{\phi_{1, j}}^{1 s}\left(T_{i}^{s}\right)=m_{\phi_{1 r, j}}^{s}\left(T_{i}^{s}\right)-m_{\phi_{1 r, j}}^{1}\left(T_{i}^{1}\right) \\
& \text { - }\left(m_{\phi_{1, j}}^{s}\left(T_{i}^{s}\right)-m_{\phi_{1, j}}^{1}\left(T_{i}^{s}\right)\right) \\
& =m_{\phi_{1 r, j}}^{1}\left(T_{i}^{1 s}\right) \\
& m_{p_{1, j}}^{1 s}\left(t_{i}\right)-m_{p_{1, r_{j}}}^{1 s}\left(T_{i}^{s}\right)=m_{p_{1, r_{j}}}^{s}\left(T_{i}^{s}\right)-m_{p_{1 r_{j}}}^{1}\left(T_{i}^{1}\right) \\
& -\left(m_{p_{1, j}}^{s}\left(T_{i}^{s}\right)-m_{p_{1, j}}^{1}\left(T_{i}^{s}\right)\right) \\
& =m_{p_{1 r j}}^{1}\left(T_{i}^{1 s}\right)
\end{aligned}
$$

As a result, the terms $\Delta m_{\phi_{1 r, j}}^{1 s}$ and $\Delta m_{p_{1 r, j}}^{1 s}$ in (38) and (39) can be formulated as follows:

$\Delta m_{\phi_{1, j}}^{1 s}\left(t_{i}\right)=E\left(\Delta G_{r \neq 1}^{s \neq 1,2}\left(T_{i}^{s \neq 1,2}\right)\right)+E\left(\Delta H_{r \neq 1}^{s \neq 1}\left(T_{i}^{s \neq 1}\right)\right)+m_{\phi_{1 r, j}}^{1}\left(T_{i}^{1 s}\right)$

$\Delta m_{p_{1 r, j}}^{1 s}\left(t_{i}\right)=E\left(\Delta G_{r \neq 1}^{s \neq 1,2}\left(T_{i}^{s \neq 1,2}\right)\right)+E\left(\Delta H_{r \neq 1}^{s \neq 1}\left(T_{i}^{s \neq 1}\right)\right)+m_{p_{1 r, j}}^{1}\left(T_{i}^{1 s}\right)$

As mentioned in the section of multipath mitigation, the expectation of $\Delta \check{\tau}_{r}$ during $T$ is not zero due to the multipath influences. This leads to non-zero expectations of $\Delta G_{r \neq 1}^{s \neq 1,2}\left(T_{i}^{s \neq 1,2}\right)$ and $\Delta H_{r \neq 1}^{s \neq 1}\left(T_{i}^{s \neq 1}\right)$. In this study, the absolute differences between $T_{i}^{1}$ and $T_{i}^{s \neq 1}$ and between $T_{i}^{2}$ and $T_{i}^{s \neq 2}$ are within $83 \mathrm{~s}$. The resulted $g_{r}^{1}\left(T_{i}^{i}\right)$ in (36) generally ranges to the level of 0.001 , and the term $E\left(\Delta H_{r \neq 1}^{s \neq 1}\left(T_{i}^{s \neq 1}\right)\right)$ is assumed to be ignorable. Since strong temporal link is applied to the ZTDs, the change of $E\left(\Delta \check{\tau}_{r}\right)$ within $T_{i}^{2 s}$ is also assumed to be small, so that $E\left(\Delta G_{r \neq 1}^{s \neq 1,2}\left(T_{i}^{s \neq 1,2}\right)\right)$ is also ignored. In this study, the satellite with the highest elevation angle at the first epoch of the processing is selected as the reference satellite, i.e., G12 for the baseline FROY-KUNU and G18 for the baseline PTHL-TOMP, respectively. Since the reference satellite with the highest elevation angle is assumed to be less influenced by the multipath effects, the multipath changes of $m_{\phi_{1 r, j}}^{1}$ and $m_{p_{1 r, j}}^{1}$ within the time interval $T_{i}^{1 s}$ are ignored.

\section{References}

Agnew DC, Larson KM (2007) Finding the repeat times of the GPS constellation. GPS Solut 11(1):71-76. https://doi.org/10.1007/ s10291-006-0038-4

Cai C, He C, Santerre R, Pan L, Cui X, Zhu J (2016) A comparative analysis of measurement noise and multipath for four constellations: GPS, BeiDou, GLONASS and Galileo. Surv Rev 48(349):287-295. https://doi.org/10.1179/1752270615Y.00000 00032
Chang XW, Yang X, Zhou T (2005) MLAMBDA: a modified LAMBDA method for integer least-squares estimation. J Geod 79(9):552-565. https://doi.org/10.1007/s00190-005-0004-х

de Jonge P, Tiberius C (1996) The LAMBDA method for integer ambiguity estimation: implementation aspects. Publication of the Delft Geodetic Computing Centre, LGR-series, no. 12. Delft University of Technology, the Netherlands. ISSN: 93-0928-2122

Elmas ZG, Aquino M, Marques HA, Monico JFG (2011) Higher order ionospheric effects in GNSS positioning in the European region. Ann Geophys 29:1383-1399. https://doi.org/10.5194/angeo $-29-1383-2011$

ESA (2014) Galileo satellite recovered and transmitting navigation signals. European Space Agency, our activities, navigation. http:// www.esa.int/Our_Activities/Navigation/Galileo_satellite_recov ered_and_transmitting_navigation_signals. Accessed 20 May 2018

ESA (2015) Sixth Galileo satellite reaches corrected orbit. European Space Agency, our activities, navigation, Galileo, Galileo Launches. http://www.esa.int/Our_Activities/Navigation/Galil eo/Galileo_Launches/Sixth_Galileo_satellite_reaches_corre cted_orbit . Accessed 20 May 2018

Euler HJ, Goad CC (1991) On optimal filtering of GPS dual frequency observations without using orbit information. Bull Geod 65(2):130-143. https://doi.org/10.1007/BF00806368

Galileo Constellation (2017) Galileo constellation information. European GNSS Service Centre. https://www.gsc-europa.eu/syste m-status/Constellation-Information. Accessed 30 Sept 2017

Geoscience Australia (2017) Final solutions of geoscience Australia. ftp://ftp.ga.gov.au/geodesy-outgoing/gnss/solutions/final I. Accessed 30 Sept 2017

Giorgi G, Teunissen PJG, Buist PJ (2008) A search and shrink approach for the baseline constrained LAMBDA method: experimental results. In: Proceedings International Symposium GPS/ GNSS, Tokyo, Japan, November 2008

Hofmann-Wellenhof B, Lichtenegger H, Wasle E (2008) GNSSglobal navigation satellite systems: GPS, GLONASS, Galileo, and more. Springer, Wien. https://doi.org/10.1007/978-3-211$73017-1$

Ifadis II (1986) The atmospheric delay of radio waves: modelling the elevation dependence on a global scale. Technical report no 38L, Chalmers University of Technology, Gothenburg

Ji SY, Chen W, Ding XL, Chen YQ, Zhao CM, Hu CW (2013) An improved cascading ambiguity resolution (CAR) method with Galileo multiple frequencies. Surv Rev 45(328):51-58. https:// doi.org/10.1179/1752270611Y.0000000021

Jonkman NF, Teunissen PJG, Joosten P, Odijk D (2000) GNSS long baseline ambiguity resolution: impact of a third navigation frequency. In: Schwarz KP (ed) Geodesy beyond 2000, IAG Symp, vol 121. Springer, Berlin, pp 349-354. https://doi. org/10.1007/978-3-642-59742-8_57

Leick A, Rapoport L, Tatarnikov D (2015) GPS satellite surveying. 4th edn. Wiley, Hoboken. (ISBN: 978-1-118-67557-1)

Li B, Verhagen S, Teunissen PJG (2014) Robustness of GNSS integer ambiguity resolution in the presence of atmospheric biases. GPS Solut 18(2):283-296. https://doi.org/10.1007/s10291-013-0329-5

Mervart L, Rocken C, Iwabuchi T, Lukes Z, Kanzaki M (2013) Precise point positioning with fast ambiguity resolution-prerequisites, algorithms and performance. In: Proc ION GNSS+ 2013, Nashville, TN, September 16-20, 2013, pp 1176-1185

Montenbruck O, Steigenberger P, Khachikyan R, Weber G, Langley RB, Mervart L, Hugentobler U (2014) IGS-MGEX: preparing the ground for multi-constellation GNSS science. Inside GNSS, January/February 2014, 42-49

Montenbruck O, Schmid R, Mercier F, Steigenberger P, Noll C, Fatkulin R, Kogure S, Ganeshan AS (2015) GNSS satellite geometry 
and attitude models. Adv Space Res 56(6):1015-1029. https://doi. org/10.1016/j.asr.2015.06.019

Montenbruck O, Steigenberger P, Prange L, Deng Z, Zhao Q, Perosanz F, Romero I, Noll C, Stürze A, Weber G, Schmid R, MacLeod K, Schaer S (2017) The multi-GNSS experiment (MGEX) of the international GNSS service (IGS) - achievements, prospects and challenges. Adv Space Res 59(7):1671-1697. https://doi. org/10.1016/j.asr.2017.01.011

Multi-GNSS Experiment (MGEX) (2017) MGEX final orbit products. NASA CDDIS. ftp://cddis.gsfc.nasa.gov/gnss/products/mgex/. Accessed June 2017

Nardo A, Li B, Teunissen PJG (2016) Partial ambiguity resolution for ground and space-based applications in a GPS + Galileo scenario: a simulation study. Adv Space Res 57(1):30-45. https://doi. org/10.1016/j.asr.2015.09.002

Ning Y, Yuan Y, Huang Z, Chai Y, Tan B (2016) A long baseline three carrier ambiguity resolution with a new ionospheric constraint. ISPRS Int J Geo Inf 5:198. https://doi.org/10.3390/ijgi5110198

Odijk D, Arora BS, Teunissen PJG (2014a) Predicting the success rate of long-baseline GPS + Galileo (partial) ambiguity resolution. J Navig 67(3):385-401. https://doi.org/10.1017/S03734633140000 $6 \mathrm{X}$

Odijk D, Teunissen PJG, Khodabandeh A (2014b) Galileo IOV RTK positioning: standalone and combined with GPS. Surv Rev 46:267-277. https://doi.org/10.1179/1752270613Y.0000000084

Odijk D, Zhang B, Khodabandeh A, Odolinski R, Teunissen PJG (2016) On the estimability of parameters in undifferenced, uncombined GNSS network and PPP-RTK user models by means of $S$-system theory. J Geod 90(1):15-44. https://doi.org/10.1007/ s00190-015-0854-9

Odijk D, Khodabandeh A, Nadarajah N, Choudhury M, Zhang B, Li W, Teunissen PJG (2017) PPP-RTK by means of S-system theory: Australian network and user demonstration. J Spat Sci 62(1):3-27. https://doi.org/10.1080/14498596.2016.1261373

RINEX (2015) RINEX, The receiver independent exchange format, version 3.03. International GNSS Service (IGS), RINEX Working Group and Radio Technical Commission for Maritime Services Special Committee 104 (RTCM-SC104), July 14, 2015

Saastamoinen J (1972) Contributions to the theory of atmospheric refraction. Bull Geod 105(1):279-298. https://doi.org/10.1007/ BF02521844

Sauer K, Vollath U, Amarillo F (2004) Three and four carriers for reliable ambiguity resolution. In: Proc ENC-GNSS 2004, Paper 022, Rotterdam, the Netherlands, May 16-19, 2004

Schaer S (1999) Mapping and predicting the earth's ionosphere using the global positioning system. Dissertation, University of Bern. Geodätisch-geophysikalische Arbeiten in der Schweiz, vol. 59, Schweizerische Geodätische Kommission, ETH Zurich

Teunissen PJG (1993) Least-squares estimation of the integer GPS ambiguities. Invited lecture, section IV theory and methodology, IAG general meeting, Beijing, August 1993

Teunissen PJG (1995) The least-squares ambiguity decorrelation adjustment: a method for fast GPS integer ambiguity estimation. J Geod 70(1-2):65-82. https://doi.org/10.1007/BF00863419

Teunissen PJG (1999) An optimality property of the integer least-squares estimator. J Geod 73(11):587-593. https://doi. org/10.1007/s001900050269

Teunissen PJG (2010) Integer least-squares theory for the GNSS Compass. J Geod 84(7):433-447. https://doi.org/10.1007/s0019 0-010-0380-8

Teunissen PJG, Khodabandeh A (2015) Review and principles of PPPRTK methods. J Geod 89(3):217-240. https://doi.org/10.1007/ s00190-014-0771-3

Teunissen PJG, Montenbruck O (eds) (2017) Springer handbook of global navigation satellite systems. Springer, Berlin. https://doi. org/10.1007/978-3-319-42928-1
Teunissen PJG, Odijk D (2003) Rank-defect integer estimation and phase-only modernized GPS ambiguity resolution. J Geod 76(910):523-535. https://doi.org/10.1007/s00190-002-0285-2

Teunissen PJG, Odijk D, Zhang B (2010) PPP-RTK: results of CORS network-based PPP with integer ambiguity resolution. J Aeronaut Astronaut Aviat Ser A 42(4):223-230

Tiberius CCJM, Pany T, Eissfeller B, de Jong K, Joosten P, Verhagen S (2002) Integral GPS-Galileo ambiguity resolution. In: Proc ENC GNSS 2002. Copenhagen, Denmark, May 2002

Wang K, Khodabandeh A, Teunissen PJG (2017) A study on predicting network corrections in PPP-RTK processing. Adv Space Res 60(7):1463-1477. https://doi.org/10.1016/j.asr.2017.06.043

Zaminpardaz S, Teunissen PJG (2017) Analysis of Galileo IOV + FOC Signals and E5 RTK performance. GPS Solut 21(4):1855-1870. https://doi.org/10.1007/s10291-017-0659-9

Zandbergen R, Dinwiddy S, Hahn J, Breeuwer E, Blonski D (2004) Galileo orbit selection. In: Proc ION GNSS 2004, Long Beach, CA, September 2004, pp 616-623

Zhang W, Cannon ME, Julien O, Alves P (2003) Investigation of combined GPS/GALILEO cascading ambiguity resolution schemes. In: Proc ION GPS/GNSS 2003, Portland, OR, September 2003, pp 2599-2610

Zhang M, Liu H, Bai Z, Qian C, Fan C, Zhou P, Shu B (2017) Fast ambiguity resolution for long-range reference station networks with ionospheric model constraint method. GPS Solut 21(2):617626. https://doi.org/10.1007/s10291-016-0551-z

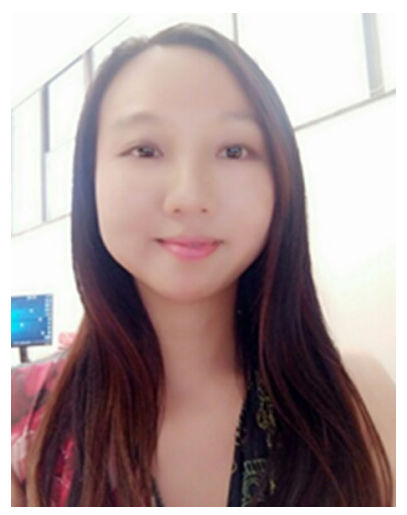

Kan Wang is a research associate in the GNSS Research Centre, Curtin University. She received her PhD in GNSS advanced modeling from ETH Zurich in 2016 , and is currently working on satellite clock modeling, multi-frequency ambiguity resolution, and multi-GNSS PPPRTK processing.

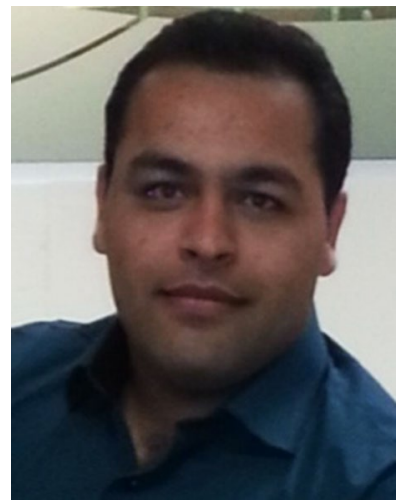

Amir Khodabandeh received his Ph.D. degree (with distinction) in Geodesy and Global Navigation Satellite Systems (GNSS) from Curtin University in 2015. $\mathrm{He}$ is currently working as a Research Fellow with the School of Civil and Enviromental Engineering, UNSW, and as a Research Associate with the GNSS Research Centre, Curtin University. He is the chair of the IAG-ICCT Joint Study Group "Multi-GNSS Theory and Algorithms" and a member of the IAG Working Group "Ionospheric and Tropospheric Impact on GNSS Positioning." His research interests include estimation theory, GNSS precise positioning, and GNSS quality control. 


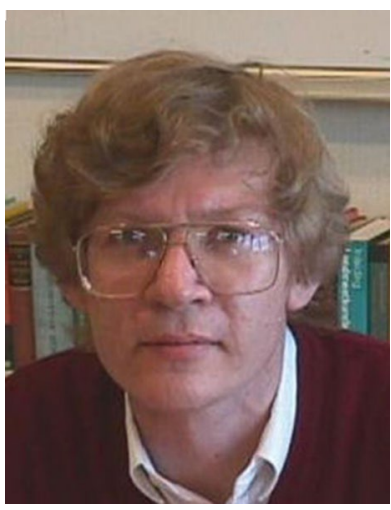

Peter J.G. Teunissen is Professor of Geodesy and Navigation at Delft University of Technology, Delft, The Netherlands, and Curtin University, Perth, Australia. $\mathrm{He}$ is recipient of an Australian
Research Council (ARC) Federation Fellowship. His current research interests are multi-GNSS and multi-frequency precise positioning, navigation and timing applications. 